Article

\title{
Evaluation of the Ability of Diet-Tracking Mobile Applications to Estimate Energy and Nutrient Intake in Japan
}

\author{
Nana Shinozaki (D) and Kentaro Murakami *(D) \\ Department of Social and Preventive Epidemiology, School of Public Health, The University of Tokyo, \\ 7-3-1 Hongo, Bunkyo-ku, Tokyo 113-0033, Japan; nana-s@m.u-tokyo.ac.jp \\ * Correspondence: kenmrkm@m.u-tokyo.ac.jp; Tel.: +81-3-5841-7872
}

Received: 1 October 2020; Accepted: 28 October 2020; Published: 29 October 2020

check for updates

\begin{abstract}
We evaluated the energy and nutrient intake estimates of popular Japanese diet-tracking mobile applications (apps). We identified five diet-tracking apps in the iTunes store during August 2020. A researcher entered the dietary data from a one-day paper-based dietary record (DR) previously obtained from apparently healthy free-living adults (15 males and 15 females; 22-65 years) into each app. The energy and nutrient intakes estimated by the apps were compared with those calculated using the Standard Tables of Food Composition in Japan based on the paper-based DR (reference method). The number of dietary variables available ranged from one (energy in Mogutan) to 17 (FiNC). Compared to the DR-based estimates, the median energy intake was significantly overestimated by MyFitnessPal, Asken, Calomiru, and Mogutan. Moreover, the intakes of many nutrients were overestimated by Asken and Calomiru and underestimated by MyFitnessPal. For energy intake, the Spearman correlation coefficient between the DR and the apps was lowest for Mogutan (0.76) and highest for FiNC (0.96). The median correlation coefficient for nutrient intakes was lower in MyFitnessPal (0.50) than in the other three apps (0.80 in Asken, 0.87 in FiNC, and 0.88 in Calomiru). These results suggest that intake calculations differ among apps. Further evaluation is needed in free-living settings, where users input their own food intake.
\end{abstract}

Keywords: mobile phone; smart phone; dietary record; dietary assessment; food composition database; portion size; Japan

\section{Introduction}

Dietary intake measurement is essential for nutritional epidemiological studies and clinical practices, including dietary counseling. Although traditional dietary assessments have relied on interviewer- or pen-and-paper-based methods, technological advances have resulted in innovative electronic approaches, such as web-based dietary records (DRs) [1-3]. Novel technology-based dietary assessment methods are considered to have the potential to reduce the burden and cost of data collection and to improve the validity and reliability of data [4-7].

In particular, with the widespread use of mobile phones, mobile applications (apps) that allow users can track their dietary intake are increasingly common [8-10]. Mobile phones have various technological features, such as wireless communication, portable design, and built-in cameras, making it possible to collect a wide range of information on people's dietary intake and to provide feedback in real time $[4,5]$. In addition, people usually have higher satisfaction and preference for dietary assessment methods using mobile phones than traditional methods [4,11-15]. Thus, the use of diet-tracking mobile apps in nutrition research may be beneficial for both participants and researchers [16].

Since there are a substantial number of apps available, selecting a high-quality, fit-for-purpose tool is a major challenge [2,17]. Ideally, diet-tracking apps for research should have a user-friendly 
interface and experience, with an extensive and reliable food composition database (FCD) [3,16,18]. Most diet-tracking apps available in app stores (commercial apps) generally have user-friendly interfaces and a large volume of FCDs [16]. However, the accuracy of the nutrient intake calculation of these apps is not necessarily guaranteed due to the minimal governmental regulation of health apps [19]. To date, there have been only a few validation studies on consumer-oriented mobile apps, such as My Meal Mate and MyFitnessPal, both showing acceptable correlation with the reference methods [20,21]. Other evaluation studies comparing the accuracy of nutritional outputs from popular diet-tracking apps reported that the ability to estimate energy and nutrient intakes differed considerably between apps, with some apps providing inaccurate values [3,19,22-25]. Evaluation of diet-tracking apps would be helpful to understand their characteristics and potential utility in nutrition research while facilitating the evidence-based selection of tools and allowing for the development and improvement of these apps. To our knowledge, however, the accuracy of dietary intake estimated by commercial diet-tracking apps has not been extensively investigated. Furthermore, research has been limited to countries outside of Asia, such as the UK [3,20], the United States [19,25], Australia $[14,24,26]$, the Netherlands [22], and Brazil [21,23].

This cross-sectional study aimed to evaluate the ability of diet-tracking mobile apps to estimate energy and nutrient intake in Japan. For the five most popular apps available in Japan, we entered food intake data previously obtained from a paper-based DR and compared the estimated dietary intakes from the apps with those calculated using the Standard Tables of Food Composition (STFCJ) in Japan based on the paper-based DR (reference method). Moreover, we investigated the energy and nutrient contents of four common foods provided by each app to assess the difference in these values in the FCDs of the apps.

\section{Materials and Methods}

\subsection{Selection of Diet-Tracking Applications}

A systematic search for mobile diet-tracking apps was conducted in the Japanese iTunes store on 6 April 2020 (Figure 1). We searched iOS apps via the iTunes search app program interface [27], which allows the use of filters and search terms to explore content within the Apple iTunes store. The search was restricted to apps available in Japanese using the filter. Each of the following terms was separately inputted into a search query in Japanese: "calorie", "diet", "nutrition", "dietary record", "food diary", "weight loss", and "body weight." These keywords were selected based on previous studies evaluating diet and nutritional apps [28-31]. The search results for each term were entered into the JavaScript Object Notation document, which was then converted and combined into an Excel file. This file includes the features of the apps, such as an app's unique identifier, an app vendor's URL, the app name, the description, the user rating, and the price [32]. Through this search process, we identified a total of 1251 apps. After removing duplicates $(n=470), 781$ unique apps were screened for eligibility.

Apps were included in this study if they (1) were free of charge or freemium (i.e., free apps with limited functionality that is unlocked by purchasing the premium version); (2) had a food logging function; (3) had the ability to calculate energy or nutrient intake; (4) had a high star rating ( $\geq 4$ out of 5) $[31,33,34]$, (5) had at least 7500 reviewers [35]; (6) had standalone functionality (operating without other programs or equipment); and (7) were available in Japanese. If the information necessary to assess eligibility was not available in the app description, the apps were installed on an iPhone 8 (iOS 13.6) to obtain the details of app features. After excluding 776 apps, the following apps were considered eligible and included in this study: FiNC, MyFitnessPal, Asken, Calomiru, and Mogutan. All five apps were downloaded and installed on the iPhone for further evaluation. 


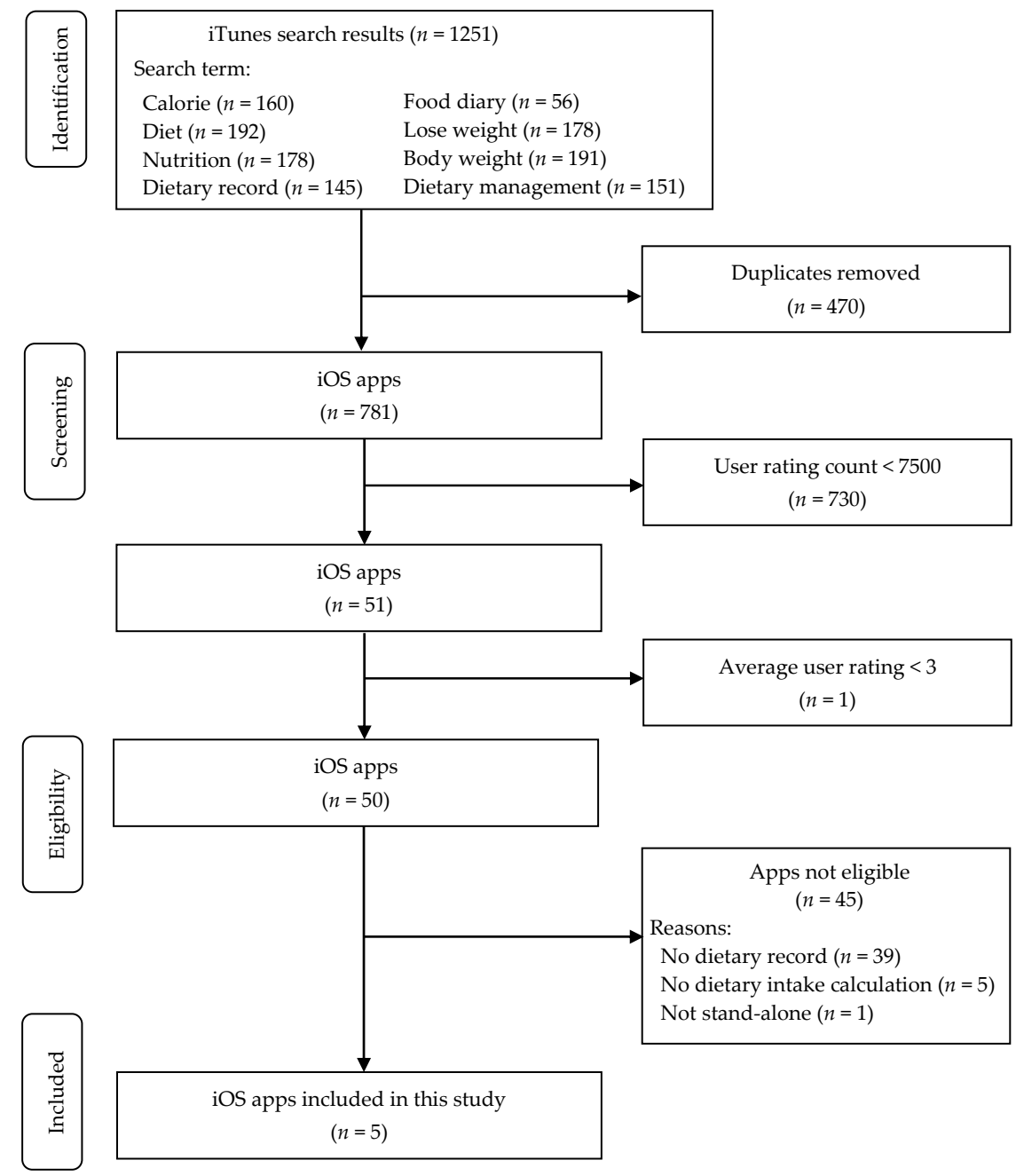

Figure 1. Flow diagram of app search and selection.

\subsection{Data Extraction}

For each app, the following characteristics were extracted from the iTunes store, app vendor's websites, and the content of each app: app name; vendor name; release date; content rating; average user rating and the number of ratings; price; language; connection with other devices; app function including passcode lock, reminders, and social networking options; and information collected about basic characteristics of users. Moreover, functions for dietary assessment were extracted, which included the following: food items available in the database, information source of the nutrient content of foods, category of eating occasions, assessment of the time of eating, input methods of food intake, methods to calculate nutrient intake, and output of dietary variables.

Furthermore, PubMed and Web of Science were searched to identify validation studies of the apps using a combination of each app name and the following string: (validity OR validation) AND (diet OR dietary OR intake OR consumption). We included studies that investigated the validity of estimated dietary intakes in comparison with a reference method such as DR. Studies were excluded if they used the website version of apps, which might differ from the mobile version, or if dietary data were manually entered by researchers into the apps. In addition, the references of the articles identified were also assessed to further identify relevant articles. We extracted the following information from eligible papers: first author, year of publication, country, participant characteristics ( $n$, gender, and age), reference method, number of times an app was used, dietary intake variables analyzed, and the results of statistical tests. 


\subsection{Comparison of Dietary Intake between a Paper-Based Dietary Record and Applications}

\subsubsection{Dietary Record Data}

A four-day weighed DR was obtained from a nationwide survey conducted in 20 study areas consisting of 23 prefectures between February and March 2013, as described elsewhere [36,37]. Briefly, the study population consisted of apparently healthy adults aged 20-69 years working at welfare facilities as well as the family members or acquaintances for those over 60 years of age. The recruitment was conducted such that each study area included two males and two females from each of the 10-year age categories (20-29, 30-39, 40-49, 50-59, and 60-69 years), resulting in invitation of 400 participants. One individual per household could participate in the survey. None of the participants were a dietitian or a medical professional, received dietary therapy by a doctor or dietitian, had a history of educational hospitalization for diabetes mellitus, or was pregnant or lactating.

Participants were asked to record all foods and beverages consumed for four nonconsecutive days (three working days and one nonworking day, excluding days before and after a night shift). Research dietitians at each welfare facility explained to the participants how to keep the DR and requested them to weigh foods and beverages using a provided digital scale or measuring spoon and cup. The research dietitians collected and checked the record sheets soon after recording and, if necessary, asked participants further information to clarify the name or amount of food on the sheet. All food codes and weights were then reconfirmed by two other research dietitians at the central office of the study.

In total, 392 individuals (196 men and 196 women) completed the study protocol. Body weight (to the nearest $0.1 \mathrm{~kg}$ ) in light clothes and body height (to the nearest $0.1 \mathrm{~cm}$ ) without shoes were measured using standardized procedures by research dietitians or medical workers. Body mass index (BMI) was calculated as body weight $(\mathrm{kg})$ divided by the square of body height $\left(\mathrm{m}^{2}\right)$. The demographic characteristics of the study participants are provided elsewhere [37,38]. Energy and nutrient intakes for individuals were calculated based on the weight of food items and their nutrient content, using the STFCJ [39]. Dietary supplements were not included in the calculation of dietary intake. The study was conducted according to the guidelines in the Declaration of Helsinki, and all procedures were approved by the Ethics Committee at the University of Tokyo, Faculty of Medicine (No. 10005).

\subsubsection{Sample Size}

The minimum sample size for assessing the difference in estimates between the DR and the apps was determined based on a previous study with a similar research question [3]. For two-tailed Wilcoxon signed-rank tests with a significance level of 0.05 , power of $80 \%$, and an effect size of 0.56 , a sample size of at least 22 was required. Thus, we selected 30 participants through stratified randomization such that three males and three females from each of the five 10-year age categories were included. For each of these participants, the DR conducted on the first day over the recording period was used for data input in the apps, considering the change in dietary intake over the recording period [40].

\subsubsection{Input of Dietary Record Data into Applications}

Food items on each participant's DR, except for drinking water and dietary supplements, were entered into each of the five apps by the first author (registered dietitian) in a uniform procedure. For branded food products and restaurant menus, the databases in the apps were searched by the names of the brand, product, or restaurant, and then, the directly matched item was selected. If there was no match, a similar generic food or dish was selected. Generic single foods were searched by the food name; if there was no directly matched food, a biologically similar food was selected. Generic mixed dishes were searched by a dish name or main food ingredients, and a dish that best represented the cooking method or food ingredients was chosen. If there was no matched dish, a dish with similar main ingredients or cooking methods was selected. To investigate the agreement between each food item selected in the app and the original food item recorded in the DR, food items were categorized 
into "closely matched foods" or "poorly matched foods". The former included food items that directly matched the items recorded in the DR, and the latter included items selected as similar foods.

The portion size of selected items in the app was adjusted for the best approximation of the net weight of that item recorded in the DR. For apps where the portion size needed to be entered as the ratio to a standard serving size (e.g., one serving), despite no information available on the standard weight, the portion size that seemed most appropriate was selected by considering the amount of food consumed. FiNC and Asken, in which several nutrients were unavailable in the free version, were upgraded to the premium version to obtain intake values. Estimated energy and nutrient intakes of each participant calculated by the apps were manually entered into Excel files, except for MyFitnessPal, which has a function for exporting data as CSV (Comma Separated Values) files.

\subsection{Assessing the Energy and Nutrient Content of Food Items in the Database of Applications}

To assess the energy and nutrient values of individual food items in the apps, four common food items were entered into each app. The energy estimates and macronutrient content (protein, total fat, and carbohydrate) were then compared with the reference values. The four items were selected from foods that existed in most of the apps and had a high consumption frequency in the 4-day DR from the entire study population $(n=392)$. The selected items were as follows: Pocky (chocolate-covered pretzel; Ezaki Glico Co., Ltd., Osaka, Japan) as an example of a branded food product, stewed chicken curry (Ichibanya Co., Ltd., Aichi, Japan) as an example of a restaurant meal, white rice as an example of a single generic food, and tonjiru (miso soup with pork and vegetables) as an example of a generic mixed dish.

If the apps showed more than one search result for each food item, a food that best represented each food and appeared at the top of the search results was selected. The reference values for Pocky and stewed chicken curry were obtained from nutrient information on the manufacturer's websites, while those for white rice and tonjiru were obtained from the STFCJ [39]. Due to a lack of information on the portion size of mixed dishes (including tonjiru) in the STFCJ, the reference value for tonjiru was calculated as the nutrient content per $100 \mathrm{~g}$ of tonjiru in the STFCJ multiplied by the mean weight of the tonjiru dishes $(311 \mathrm{~g})$ recorded in the 4-day DR obtained from the entire study population.

\subsection{Data Analysis}

To describe each app, we tabulated the basic and nutrition-related features and the results of the validation studies. Moreover, the percentage of food items categorized into "closely matched foods" was calculated for each app. Energy and nutrient intakes estimated by each app were compared with those estimated from the DR using the STFCJ as a reference. Although trans fatty acid intake was calculated by MyFitnessPal, the STFCJ did not have the analytical value of trans fatty acids in foods. In addition, some apps offered to calculate sugar intake, while the type of sugar that the app estimated (total sugar, added sugar, or free sugar [41]) was not clear. Therefore, these two nutrients estimated from the apps were excluded from the comparison with the DR.

To assess the ability to estimate absolute intake among the apps, we compared mean and median intakes between the apps and the DR using paired $t$-test and Wilcoxon signed-rank test, respectively. Since both showed similar results, the results of the median comparison are shown here. Next, the ranking ability of the apps was evaluated using Spearman correlation coefficients with DR-based estimates. To summarize the correlation coefficients for each nutrient, we calculated the median value of the correlation coefficient of nutrients for each app. Additionally, a Bland-Altman plot $[42,43]$ was used to assess the agreement of energy and macronutrient intakes (protein, total fat, and carbohydrate) estimated using the apps and those estimated by the DR. The upper and lower limits of agreement were calculated as the mean difference \pm 1.96 standard deviations (SD). The proportional error between the two methods was evaluated using regression analysis.

Finally, we counted the number of search results for four common food items and calculated the relative differences of energy and macronutrients estimated by the apps from the reference values. 
The relative difference was calculated as follows: relative difference $(\%)=(($ app - reference $) /$ reference $)$ $\times 100$. The statistical software package SAS version 9.4 was used for the analyses. Two-sided $p$ values $<0.05$ were considered statistically significant.

\section{Results}

\subsection{Characteristics of the Selected Diet-Tracking Applications}

There was some variation of features between the apps (Table S1). All the apps had a selection of functions available only for the paid version, except for the Mogutan app. Most apps were available only in one or two languages, except for MyFitnessPal, which was originally developed in the US and supported 20 languages. The app features related to dietary assessment are shown in Table 1. General foods and mixed dishes were included in the database of all the apps, whereas restaurant meals or branded food products were not covered in the Mogutan app. The number of food items in the database was largest in MyFitnessPal $(n \geq 4,000,000)$ and smallest in Mogutan $(n=278)$. For FCDs, MyFitnessPal, Asken, and Calomiru used their own estimated values and the nutrient data reported by food manufacturers. MyFitnessPal and Asken also use nutrient data from the national nutrient databases of the United States and Japan, respectively. Moreover, MyFitnessPal has a crowd-sourced database, making it possible for users to upload food entries. Foods are mainly selected using the text search of the databases, while items are registered by selecting food stickers in the Mogutan app. The amount of food consumed is adjusted by entering the percentage to the standard serving size in most apps, while it is adjusted by selecting a portion size from three categories in Mogutan. In addition, the amount of food can be directly entered into the MyFitnessPal app. The maximum number of dietary variables available, including the premium version, ranged from one (energy in Mogutan) to 17 (FiNC). Two validation studies [21,44] were identified for MyFitnessPal (Table S2). Compared to paper-based DRs or 24-h dietary recalls, underestimation of nutrients by the app was observed in both studies.

\subsection{Comparison of Dietary Intake between the Applications and the Dietary Record}

The percentage of food items classified as closely matched foods was the highest in the FiNC (95\%) app, followed by MyFitnessPal (93\%), Asken (92\%), Calomiru (88\%), and Mogutan (57\%). Table 2 shows the median intakes of energy and nutrients estimated by the DR and those estimated by the five apps in the thirty participants (mean age, 44.5 (SD: 13.4, range: 22-65) years; mean BMI, 22.7 (SD: 3.0$) \mathrm{kg} / \mathrm{m}^{2}$ ). Energy intake was significantly overestimated by MyFitnessPal, Asken, Calomiru, and Mogutan compared with that estimated by the DR. Among the apps providing nutrient intakes, FiNC had the lowest number of nutrients that significantly differed between the DR and the apps (only for niacin intake). MyFitnessPal significantly underestimated saturated fatty acids, monounsaturated fatty acids, polyunsaturated fatty acids, cholesterol, total dietary fiber, sodium, and potassium. On the other hand, Asken overestimated the intake of many nutrients, including carbohydrates, total dietary fiber, sodium, calcium, iron, and vitamin D. Similarly, Calomiru overestimated the intake of protein, carbohydrates, and sodium. 
Table 1. Nutritional functionalities of the five diet-tracking applications included in this study ${ }^{\text {a }}$

\begin{tabular}{|c|c|c|c|c|c|}
\hline Function & FiNC & MyFitnessPal & Asken & Calomiru & Mogutan \\
\hline \multicolumn{6}{|l|}{ Food items available in the database } \\
\hline General foods and dishes & $\checkmark$ & $\checkmark$ & $\checkmark$ & $\checkmark$ & $\checkmark$ \\
\hline Restaurant meals & $\checkmark$ & $\checkmark$ & $\checkmark$ & $\checkmark$ & None \\
\hline Branded food products & $\checkmark$ & $\checkmark$ & $\checkmark$ & & Only four items \\
\hline Number of food items & No information & $\geq 4,000,000$ & $\geq 100,000$ & $\begin{array}{l}\text { About } 4000 \text { for general } \\
\text { foods/about } 15,000 \text { for } \\
\text { restaurant meals and branded } \\
\text { food products }\end{array}$ & 278 \\
\hline \multicolumn{6}{|l|}{ Sources of nutrient content of foods } \\
\hline Food manufacturers & No information & $\checkmark$ & $\checkmark$ & $\checkmark$ & No information \\
\hline Estimation by application vendors & No information & $\checkmark$ & $\checkmark$ & $\checkmark$ & No information \\
\hline National nutrient databases & No information & $\begin{array}{l}\text { USDA SR } \\
\text { Crowd-sourced database }{ }^{b}\end{array}$ & STFCJ 2015 & Not specified $^{c}$ & No information \\
\hline User-generated data & None & & None & None & None \\
\hline \multicolumn{6}{|l|}{ Input of eating occasion } \\
\hline Eating occasion category & Breakfast/lunch/dinner/snack & Customizable up to six categories & Breakfast/lunch/dinner/snack & Breakfast/lunch/dinner/snack & Breakfast/lunch/dinner/snack \\
\hline Time of eating & $\checkmark$ & $\checkmark$ & $\checkmark$ & $\checkmark$ & None \\
\hline $\begin{array}{l}\text { Input methods of food intake } \\
\text { Food images }\end{array}$ & $\checkmark$ & None & $\triangleleft^{\mathrm{d}}$ & $\checkmark$ & None \\
\hline Text search from food databases & $\checkmark$ & $\checkmark$ & $\checkmark$ & $\checkmark$ & None \\
\hline Barcode scanner & None & $\checkmark$ & None & None & None \\
\hline Original recipes or foods & None & $\checkmark$ & None & $\checkmark$ & $\checkmark$ \\
\hline Other & None & None & None & None & Select from food stickers \\
\hline Quantification of food intake & $\begin{array}{l}\text { Percentage to standard serving sizes } \\
\text { (unit: } 10 \% \text { ) }\end{array}$ & $\begin{array}{l}\text { Percentage to standard serving } \\
\text { sizes (unit: } 1 \% \text { ) or amount } \\
\text { (gram/milliliter/cup/ounce) }\end{array}$ & $\begin{array}{l}\text { Percentage to standard serving } \\
\text { sizes (unit: any percentages) or } \\
\text { energy content }\end{array}$ & $\begin{array}{l}\text { Percentage to standard serving } \\
\text { sizes (unit: } 1 \% \text { ) }\end{array}$ & $\begin{array}{l}\text { Three PS categories: all/half/c } \\
\text { little or energy content }\end{array}$ \\
\hline \multicolumn{6}{|l|}{ Methods to calculate nutrient intake } \\
\hline Manual calculation by dietitians & None & None & None & $\checkmark$ & None \\
\hline Automated calculation from inputted food intake & $\checkmark$ & $\checkmark$ & $\checkmark$ & $\checkmark$ & $\checkmark$ \\
\hline Semiautomatic image analysis & $\checkmark$ & None & None & $\checkmark$ & None \\
\hline Output of dietary variables (shown as intake values) & Energy and 15 nutrients (+sugar for & Energy and 12 nutrients & Energy ( +13 nutrients for the & Energy and 5 nutrients & Energy \\
\hline Validation studies & None & Two studies & None & None & None \\
\hline
\end{tabular}

PS, portion size; STFCJ, Standard Tables of Food Composition in Japan; USDA SR, US Department of Agriculture National Nutrient Database for Standard Reference. ${ }^{a}$ Functions are for
the free version of each app unless otherwise indicated. The check mark represents that apps have the respective features. ${ }^{b}$ Users can freely upload food items and correct the energy and $^{\text {and }}$. the free version of each app unless otherwise indicated. The check mark represents that apps have the respective features. ${ }^{b}$ Users can freely upload food items and correct the
nutrient content. c Referred to as "food composition databases" (no further information). ${ }^{d}$ Users can register food photos as memos although the photos are not analyzed. 
Table 2. Median intakes of energy and nutrients estimated by a one-day paper-based dietary record (DR) and those estimated by the five diet-tracking applications among Japanese adults $(n=30)$.

\begin{tabular}{|c|c|c|c|c|c|c|c|c|c|c|c|c|c|c|c|c|c|c|c|c|c|c|c|}
\hline \multirow[b]{2}{*}{ Variables } & \multicolumn{3}{|c|}{ Paper-based DR ${ }^{a}$} & \multicolumn{4}{|c|}{ FiNC $^{b, c}$} & \multicolumn{4}{|c|}{ MyFitnessPal $^{b}$} & \multicolumn{4}{|c|}{ Asken b,d } & \multicolumn{4}{|c|}{ Calomiru ${ }^{b}$} & \multicolumn{4}{|c|}{ Mogutan $^{b}$} \\
\hline & Median & $P_{25}$ & $P_{75}$ & Median & $P_{25}$ & $P_{75}$ & $P^{\mathrm{e}}$ & Median & $P_{25}$ & $P_{75}$ & $P^{\mathrm{e}}$ & Median & $P_{25}$ & $P_{75}$ & $P^{\mathrm{e}}$ & Median & $P_{25}$ & $P_{75}$ & $P^{\mathrm{e}}$ & Median & $\mathrm{P}_{25}$ & $P_{75}$ & $P^{\mathrm{e}}$ \\
\hline Energy (kJ/day) & 8556 & 6514 & 10,095 & 8512 & 6853 & 10,887 & 0.77 & 10,170 & 6936 & 11784 & 0.003 & 9065 & 6732 & 11,891 & 0.02 & 9010 & 7376 & 11,803 & 0.001 & 9525 & 7092 & 11,724 & 0.004 \\
\hline Protein (g/day) & 69.2 & 61.7 & 80.9 & 72.5 & 56.0 & 83.0 & 0.83 & 65.3 & 53.3 & 86.2 & 0.88 & 75.2 & 56.6 & 97.9 & 0.06 & 74.8 & 62.6 & 100.0 & 0.0004 & - & - & - & \\
\hline Total fat (g/day) & 59.1 & 34.2 & 74.8 & 58.0 & 39.0 & 88.0 & 0.33 & 56.1 & 39.9 & 79.9 & 0.46 & 61.0 & 42.6 & 80.4 & 0.09 & 60.7 & 38.3 & 85.9 & 0.06 & - & - & - & - \\
\hline Saturated fatty acid (g/day) & 14.6 & 9.6 & 23.3 & - & - & - & - & 2.1 & 0.4 & 6.0 & $<0.0001$ & 17.1 & 11.0 & 23.8 & 0.99 & - & - & - & - & - & - & - & - \\
\hline Monounsaturated fatty acid ( $\mathrm{g} /$ day) & 20.9 & 11.9 & 27.8 & - & - & - & - & 2.7 & 0.0 & 4.7 & $<0.0001$ & - & - & - & - & - & - & - & - & - & - & - & - \\
\hline Polyunsaturated fatty acid (g/day) & 10.9 & 7.7 & 14.3 & - & - & - & - & 1.6 & 0.4 & 3.3 & $<0.0001$ & - & - & - & - & - & - & - & - & - & - & - & - \\
\hline Trans fatty acid (g/day) & - & - & & - & - & - & - & 0.0 & 0.0 & 0.0 & - & - & - & - & - & - & - & - & - & - & - & - & - \\
\hline Cholesterol (mg/day) & 338 & 219 & 549 & - & - & - & - & 8 & 0 & 23 & $<0.0001$ & - & - & - & - & - & - & - & - & - & - & - & - \\
\hline Carbohydrate (g/c & 274.3 & 207.5 & 338.0 & 267.5 & 209.0 & 342.0 & 0.74 & 282.3 & 192.7 & 345.8 & 0.45 & 292.4 & 207.1 & 346.9 & 0.03 & 304.7 & 202.8 & 354.7 & 0.008 & - & - & - & - \\
\hline $\operatorname{Sugar}^{\mathrm{f}}(\mathrm{g} /$ day $)$ & - & - & - & 254.5 & 199.0 & 326.0 & - & 5.5 & 0.0 & 16.9 & - & - & - & - & - & 291.2 & 196.2 & 342.6 & - & - & - & - & - \\
\hline Total dietary fiber (g/day) & 12.7 & 8.4 & 16.2 & 12.5 & 9.0 & 16.0 & 0.24 & 6.8 & 3.1 & 10.5 & 0.0002 & 20.5 & 10.6 & 25.5 & $<0.0001$ & 13.1 & 9.4 & 16.8 & 0.10 & - & - & - & - \\
\hline Sodium $\mathrm{g}(\mathrm{mg} /$ day $)$ & 3994 & 2700 & 4644 & 3780 & 2953 & 4803 & 0.64 & 2893 & 2039 & 4215 & 0.03 & 4213 & 3346 & 5591 & 0.004 & 4750 & 3839 & 5264 & $<0.0001$ & - & - & - & - \\
\hline Potassium (mg/day) & 2484 & 2032 & 2981 & 2501 & 2026 & 2945 & 0.46 & 1033 & 331 & 1580 & $<0.0001$ & - & - & - & - & - & - & - & - & - & - & - & - \\
\hline Calcium $(\mathrm{mg} / \mathrm{d}$ & 447 & 312 & 664 & 437 & 320 & 720 & 0.46 & $-\mathrm{h}$ & - & - & - & 501 & 389 & 640 & 0.002 & - & - & - & - & - & - & - & - \\
\hline Magnesium (mg/day) & 270 & 227 & 314 & 274 & 214 & 330 & 0.45 & - & - & - & - & - & - & - & - & - & - & - & - & - & - & - & - \\
\hline Iron (mg/day) & 7.3 & 5.6 & 8.8 & 7.4 & 6.0 & 9.3 & 0.20 & $-\mathrm{h}$ & - & - & - & 8.2 & 5.9 & 10.2 & 0.03 & - & - & - & - & - & - & - & - \\
\hline Vitamin $\mathrm{A}^{\mathrm{i}}(\mu \mathrm{g} /$ day $)$ & 391 & 255 & 628 & - & - & - & - & $-\mathrm{h}$ & - & - & - & 450 & 220 & 807 & 0.07 & - & - & - & - & - & - & - & - \\
\hline Vitamin $\mathrm{D}(\mu \mathrm{g}$ & 4.0 & 1.7 & 8.4 & 2.6 & 1.5 & 5.6 & 0.07 & - & - & - & - & - & - & & & - & - & - & - & - & - & - & - \\
\hline$\alpha$-Tocopherol (mg/day) & 6.4 & 4.0 & 8.3 & & & - & & - & - & - & - & 8.6 & 5.6 & 11.0 & 0.0001 & - & - & - & - & - & - & - & - \\
\hline Thiamin (mg/day) & 0.95 & 0.64 & 1.32 & 1.00 & 0.70 & 1.60 & 0.1 & - & - & - & - & 1.03 & 0.64 & 1.33 & 0.23 & - & - & - & - & - & - & - & - \\
\hline Riboflavin (mg/day) & 1.24 & 1.02 & 1.51 & 1.40 & 0.90 & 1.70 & 0.33 & - & - & - & - & 1.42 & 0.97 & 1.66 & 0.86 & - & - & - & - & - & - & - & - \\
\hline Niacin (mg/day) & 17.7 & 13.0 & 21.5 & 32.5 & 23.0 & 39.0 & $<0.0001$ & - & - & - & - & - & - & - & - & - & - & - & - & - & - & - & - \\
\hline Vitamin B-12 ( $\mu \mathrm{g} /$ day) & 3.3 & 2.6 & 7.0 & 4.9 & 2.9 & 9.0 & 0.06 & - & - & - & - & - & - & - & - & - & - & - & - & - & - & - & - \\
\hline Vitamin C (mg/day) & 81 & 44 & 121 & 94 & 48 & 130 & 0.35 & $-\mathrm{h}$ & - & - & - & 90 & 49 & 125 & 0.27 & - & - & - & - & - & - & - & - \\
\hline
\end{tabular}

DR, dietary record; $P_{25}$, 25th percentile; $P_{75}$, 75th percentile. ${ }^{a}$ Estimated based on a one-day paper-based DR using the Standard Tables of Food Composition in Japan [39]. ${ }^{\mathrm{b}}$ Estimated

from the apps by entering the same food items on the DR (except for dietary supplements and drinking water) into each app. ${ }^{c}$ Sugar intake is available for the premium version only. ${ }^{d}$ Intake values of all nutrients (excluding energy) were available only for the premium version. ${ }^{e}$ The difference from values derived from the DR was tested by the Wilcoxon signed-rank test. ${ }^{\mathrm{f}}$ The type of sugar that the app estimated was not clearly defined for FiNC or MyFitnessPal. For Calomiru, sugar intake is basically calculated as carbohydrate intake (g) minus the dietary fiber intake $(\mathrm{g})$. ${ }^{\mathrm{g}}$ For comparison, salt intakes estimated by FiNC, Asken, and Calomiru were converted to sodium intake as follows: sodium $(\mathrm{mg})=$ salt $(\mathrm{mg}) / 2.54 \times 1000$ [45].

${ }^{\mathrm{h}}$ Only the percentages to the dietary reference intakes of the dietary guidelines for Americans are shown in MyFitnessPal. ${ }^{\mathrm{i}}$ Retinol activity equivalent. 
Spearman correlation coefficients for estimates between the DR and the apps are shown in Table 3. The correlation coefficient for energy was the lowest for Mogutan (0.76) and the highest for FiNC (0.96). Although the number of nutrients estimated differed among the apps, the median correlation coefficient for nutrients was lower in MyFitnessPal (0.50) than in the other three apps (0.80 in Asken, 0.87 in FiNC, and 0.88 in Calomiru).

Table 3. Spearman correlation coefficients for the association between energy and nutrient intakes estimated by a one-day dietary record and those estimated by the five diet-tracking applications among Japanese adults $(n=30)^{\text {a }}$.

\begin{tabular}{|c|c|c|c|c|c|}
\hline & FiNC & MyFitnessPal & Asken & Calomiru & Mogutan \\
\hline Variables & $r$ & $r$ & $r$ & $r$ & $r$ \\
\hline Energy (kJ/day) & 0.96 & 0.90 & 0.95 & 0.93 & 0.76 \\
\hline Protein (g/day) & 0.93 & 0.74 & 0.84 & 0.88 & - \\
\hline Total fat (g/day) & 0.87 & 0.81 & 0.89 & 0.88 & - \\
\hline Saturated fatty acid (g/day) & - & 0.26 & 0.86 & - & - \\
\hline Monounsaturated fatty acid (g/day) & - & 0.33 & - & - & - \\
\hline Polyunsaturated fatty acid (g/day) & - & 0.49 & - & - & - \\
\hline Cholesterol (mg/day) & - & 0.23 & - & - & - \\
\hline Carbohydrate (g/day) & 0.95 & 0.82 & 0.95 & 0.92 & - \\
\hline Total dietary fiber (g/day) & 0.93 & 0.55 & 0.89 & 0.85 & - \\
\hline Sodium (mg/day) & 0.81 & 0.47 & 0.73 & 0.76 & - \\
\hline Potassium (mg/day) & 0.92 & 0.51 & - & - & - \\
\hline Calcium (mg/day) & 0.92 & - & 0.84 & - & - \\
\hline Magnesium (mg/day) & 0.91 & - & - & - & - \\
\hline Iron (mg/day) & 0.85 & - & 0.80 & - & - \\
\hline Vitamin $\mathrm{A}^{\mathrm{b}}(\mu \mathrm{g} /$ day $)$ & - & - & 0.65 & - & - \\
\hline Vitamin D ( $\mu \mathrm{g} /$ day) & 0.92 & - & - & - & - \\
\hline$\alpha$-Tocopherol (mg/day) & - & - & 0.76 & - & - \\
\hline Thiamin (mg/day) & 0.67 & - & 0.72 & - & - \\
\hline Riboflavin (mg/day) & 0.71 & - & 0.55 & - & - \\
\hline Niacin (mg/day) & 0.82 & - & - & - & - \\
\hline Vitamin B-12 ( $\mu \mathrm{g} /$ day) & 0.70 & - & - & - & - \\
\hline Vitamin C (mg/day) & 0.73 & - & 0.75 & - & - \\
\hline
\end{tabular}

${ }^{\text {a }}$ All correlation coefficients were significantly higher than $0(p<0.05)$ except for that for saturated fatty acid $(p=0.17)$, monounsaturated fatty acid $(p=0.08)$, and cholesterol $(p=0.21)$ in MyFitnessPal. ${ }^{\mathrm{b}}$ Retinol activity equivalent.

Bland-Altman plots used to assess the agreement of intakes between the DR and the apps are shown in Figure 2 (for energy) and Figures S1-S3 (for protein, total fat, and carbohydrate, respectively). For energy, MyFitnessPal and Mogutan had greater mean differences and wider limits of agreements. For all the macronutrients (plotted except for Mogutan), the mean differences were the largest in Calomiru and the limits of agreement were the widest in MyFitnessPal. Regression analysis showed significant linear trends in protein intake estimated by FiNC $(\beta=0.19, p=0.01)$ and Calomiru $(\beta=0.38$, $p=0.001)$. 


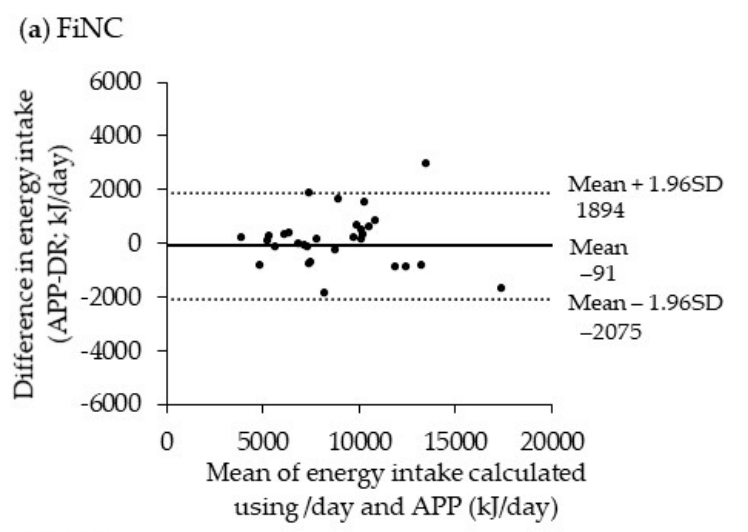

(b) MyFitnessPal
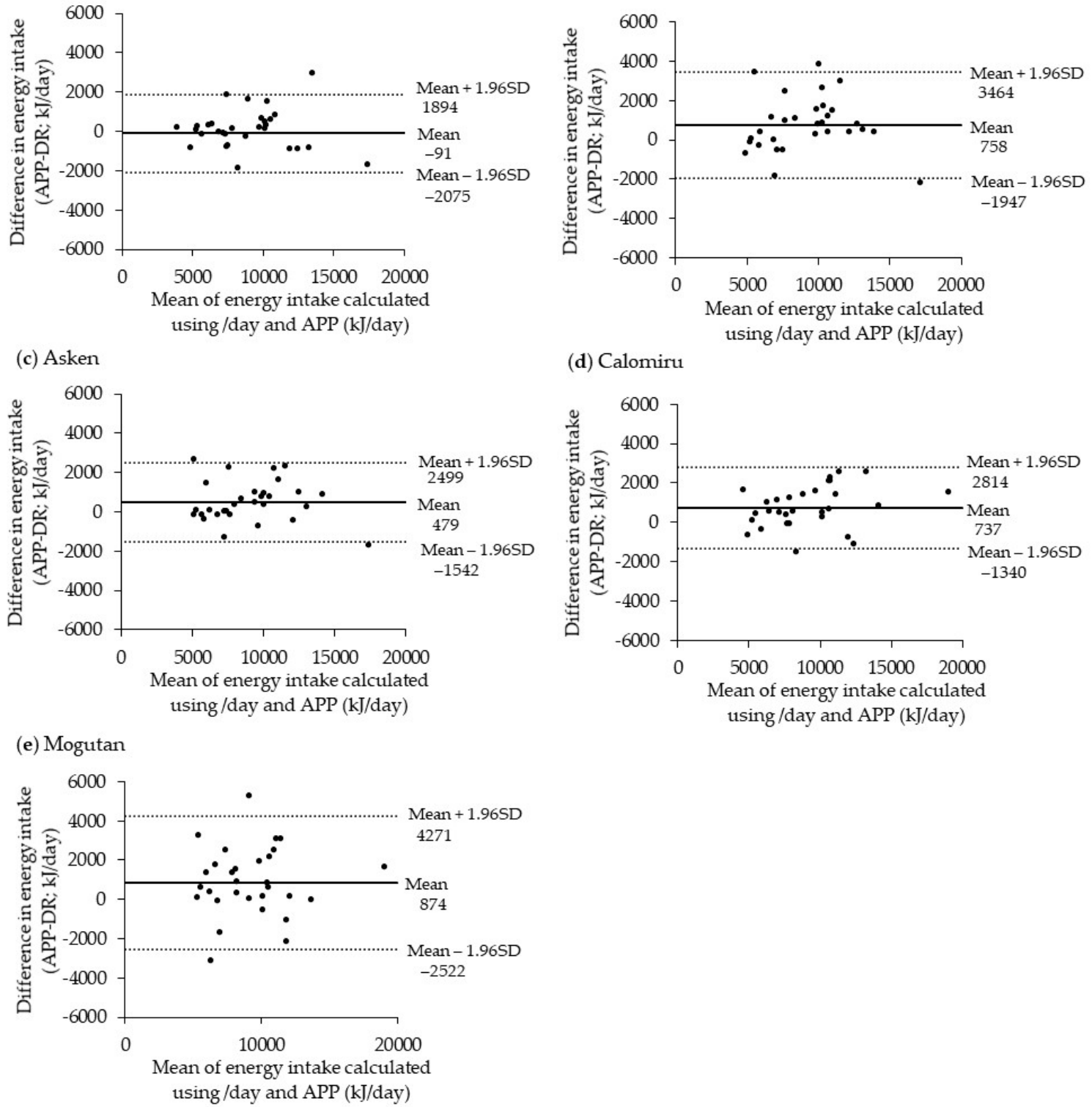

Figure 2. Bland-Altman plots assessing the agreement of the estimated energy intake between a paper-based dietary record (DR) and each application (APP) in 30 Japanese adults: (a) FiNC, (b) MyFitnessPal, (c) Asken, (d) Calomiru, and (e) Mogutan. The solid line represents the mean difference, and the dotted line represents lower and upper $95 \%$ limits of agreement.

\subsection{Energy and Nutrient Content of Four Food Items}

The energy and nutrient contents of the four food items are shown in Table 4. The number of search results was the largest in MyFitnessPal for three of the four items. Moreover, Mogutan showed large differences in energy content from the reference value for all items. For Pocky and stewed chicken curry, the energy and macronutrient contents in FiNC and Asken were not consistent with the reference value. For white rice, MyFitnessPal overestimated energy and nutrient contents, up to a maximum of $200 \%$ difference (total fat). FiNC showed $0-4 \%$ relative differences in energy and nutrient contents, since the amount of rice could not be adjusted to $100 \mathrm{~g}$ using FiNC, which employed portion size adjustments in units of $10 \%$. The energy and nutrient content of tonjiru was overestimated by all the apps, with a range of $61-264 \%$. 
Table 4. Examples of energy and nutrient contents in the diet-tracking applications for four food items ${ }^{\text {a }}$.

\begin{tabular}{|c|c|c|c|c|c|c|c|c|c|c|c|}
\hline \multirow[b]{3}{*}{ Food items } & \multirow[b]{3}{*}{ Reference $^{b}$} & \multicolumn{10}{|c|}{ Dietary Tracking Applications } \\
\hline & & \multicolumn{2}{|r|}{ FiNC } & \multicolumn{2}{|c|}{ MyFitnessPal } & \multicolumn{2}{|r|}{ Asken } & \multicolumn{2}{|c|}{ Calomiru } & \multicolumn{2}{|c|}{ Mogutan } \\
\hline & & Value & $\begin{array}{c}\text { Relative } \\
\text { Difference }(\%)^{\mathrm{c}}\end{array}$ & Value & $\begin{array}{c}\text { Relative } \\
\text { Difference }(\%)^{\mathrm{c}}\end{array}$ & Value & $\begin{array}{c}\text { Relative } \\
\text { Difference }(\%)^{c}\end{array}$ & Value & $\begin{array}{c}\text { Relative } \\
\text { Difference }(\%)^{\mathrm{c}}\end{array}$ & Value & $\begin{array}{c}\text { Relative } \\
\text { Difference }(\%)^{\mathrm{c}}\end{array}$ \\
\hline \multicolumn{12}{|l|}{ Pocky Chocolate, Glico, 1 pack } \\
\hline Number of search results $d$ & - & 2 & - & $>500$ & - & 70 & - & 3 & - & NA & - \\
\hline Energy (kJ) & 761 & 732 & -4 & 761 & 0 & 732 & -4 & 761 & 0 & 318 & -58 \\
\hline Protein (g) & 3.0 & 3.1 & 3 & 3.0 & 0 & 3.1 & 3 & 3.1 & 3 & - & - \\
\hline Total Fat (g) & 8.2 & 7.6 & -7 & 8.2 & 0 & 7.6 & -7 & 8.2 & 0 & - & - \\
\hline Carbohydrate (g) & 24.0 & 23.5 & -2 & 24.0 & 0 & 23.5 & -2 & 23.9 & 0 & - & - \\
\hline \multicolumn{12}{|c|}{ Stewed chicken curry (pork source), CURRY HOUSE CoCo ICHIBANYA, 1 serving } \\
\hline Number of search results $\mathrm{e}$ & - & 2 & - & 22 & - & 3 & - & 2 & - & NA & - \\
\hline Energy $(\mathrm{kJ})$ & 3661 & 3561 & -3 & 3661 & 0 & 3561 & -3 & 3661 & 0 & $2720^{\mathrm{f}}$ & $-26^{\mathrm{f}}$ \\
\hline Protein $(\mathrm{g})$ & 22.7 & 24.2 & 6 & 22.7 & 0 & 24.2 & 7 & 22.7 & 0 & - & - \\
\hline Total Fat (g) & 28.3 & 25.2 & -11 & 28.3 & 0 & 25.2 & -11 & 28.3 & 0 & - & - \\
\hline Carbohydrate (g) & 126.7 & 125.4 & -1 & 126.7 & 0 & 125.4 & -1 & 126.7 & 0 & - & - \\
\hline \multicolumn{12}{|l|}{ White rice, cooked, $100 \mathrm{~g}$} \\
\hline Number of search results $g$ & - & 35 & - & 432 & - & 26 & - & $>500$ & - & NA & - \\
\hline Energy (kJ) & 703 & $728^{\mathrm{h}}$ & 4 & 1063 & 51 & 703 & 0 & 698 & -1 & 983 & 40 \\
\hline Protein $(\mathrm{g})$ & 2.5 & $2.6^{\mathrm{h}}$ & 4 & 6.1 & 144 & 2.5 & 0 & 2.5 & 0 & - & - \\
\hline Total Fat (g) & 0.3 & $0.3^{\mathrm{h}}$ & 0 & 0.9 & 200 & 0.3 & 0 & 0.3 & 0 & - & - \\
\hline Carbohydrate (g) & 37.1 & $38.6^{\mathrm{h}}$ & 4 & 77.1 & 108 & 37.1 & 0 & 37.1 & 0 & - & - \\
\hline \multicolumn{12}{|c|}{ Tonjiru (miso soup with pork and vegetables), 1 serving } \\
\hline Number of search results $\mathrm{i}^{\mathrm{i}}$ & - & 163 & - & $>500$ & - & 104 & - & 237 & - & NA & - \\
\hline Energy (kJ) & 351 & 849 & 142 & 1100 & 213 & 611 & 74 & 841 & 139 & 1059 & 201 \\
\hline Protein $(\mathrm{g})$ & 4.7 & 10.6 & 127 & 12.6 & 170 & 9.1 & 95 & 9.4 & 102 & - & - \\
\hline Total Fat (g) & 4.7 & 11.9 & 155 & 17.0 & 264 & 7.5 & 61 & 12.6 & 170 & - & - \\
\hline Carbohydrate (g) & 6.2 & 12.7 & 104 & 15.2 & 144 & 10.3 & 66 & 11.9 & 91 & - & - \\
\hline
\end{tabular}

NA, not applicable. ${ }^{a}$ Energy and nutrient contents are shown for one food item that best represented each food in the search results in each app. ${ }^{\mathrm{b}}$ The reference values for Pocky and stewed chicken curry were obtained from nutrient information on the manufacturer's websites. The reference values for white rice were derived from food code 1088, "rice, short grain, paddy rice, nonglutinous rice, well-milled, meshi (cooked rice)" in the Standard Tables of Food Composition in Japan (STFCJ) [39]. The reference values for tonjiru were calculated as the nutrient content per $100 \mathrm{~g}$ of tonjiru in the STFCJ multiplied by the mean weight of 313 tonjiru dishes $(311 \mathrm{~g})$ recorded in the 4-day DR obtained from the entire study population (n 392). ${ }^{c}$ Relative difference $(\%)=(($ app - reference $) /$ reference $) \times 100 .{ }^{\mathrm{d}}$ Apps were searched using the keywords "Pocky chocolate" and "Glico". e Apps were searched using the keywords "chicken nikomi curry" (menu name in Japanese) and "CoCo ICHIBANYA". f The same product was not identified. Instead, the energy content of generic curry rice was shown. g Apps were searched using the keyword "hakumai" (white rice in Japanese). $\mathrm{h}$ The energy and nutrient values were shown for $104 \mathrm{~g}$ of white rice because the position size could not be adjusted to $100 \mathrm{~g} .{ }^{i}$ Apps were searched using the keywords "tonjiru" and "butajiru". 


\section{Discussion}

\subsection{Summary of Results}

In this study, we described the features of five popular diet-tracking mobile apps in Japan and evaluated their ability to estimate energy and nutrient intake. Consistent with previous studies $[3,19,22-25]$, there were vast differences in estimated intakes among the apps. FiNC could adequately estimate energy and nutrient intake both at the group and individual levels and could rank individuals according to their intakes.

In comparison, these features were poor in MyFitnessPal and Mogutan. Although Asken and Calomiru had moderate ranking capabilities, they demonstrated inadequacy regarding the estimation of energy and nutrient intakes at the group level. Moreover, Calomiru was associated with a poor ability to estimate energy and nutrient intakes at the individual level. This may be attributable to the difference in the functions of apps relevant to nutrient intake calculation, such as FCDs used for estimating dietary intakes, methods to search and select food items, and portion size estimation. Moreover, only MyFitnessPal had been previously validated, indicating the lack of scientific evidence in most of the apps. To our knowledge, this is the first study to evaluate the ability of commercial diet-tracking mobile apps to estimate energy and nutrient intake in Japan.

\subsection{Food Composition Databases}

The extensiveness of food selections in FCDs may affect the efficacy of users in the selection of food items that exactly match the foods they consume. The number of food items in the database was the smallest in Mogutan, which did not include restaurant or brand-name food products. Consequently, Mogutan had the lowest percentage of food items that matched food items in the DR, resulting in reduced ability to estimate energy intake compared to other apps. On the other hand, MyFitnessPal, which used the FCD of the US and a crowd-sourced database, had the greatest number of food selections, including restaurant and brand-name foods, and a high percentage of food items matching foods in the DR. Nevertheless, MyFitnessPal had a poor ability to estimate energy and nutrient intake both at the group and individual levels. This paradox may be due to the fact that the US-based FCD may differ in food selections and nutrient content in foods when compared to Japanese databases [25]. Moreover, although the crowd-sourced database is potentially beneficial for enriching the data, given that users can upload food items by entering only the food name and energy content, some nutrient values may be incorrect or missing, especially for foods that are not labeled [3,16,19,21,22]. Indeed, MyFitnessPal underestimated the intake of nutrients that are not required for food labels in Japan [46], including fatty acids, cholesterol, total dietary fiber, sodium, and potassium. The underestimation of nutrients by MyFitnessPal was also reported in two validation studies [21,44]. Although users can correct inaccurate values, the efficacy of such user-based quality control remains unclear [22].

Although FiNC and Asken used the manufacturer's data for the FCDs, we found that the energy and nutrient values of two commercial foods (Pocky and stewed chicken curry) were not consistent with the reference values. Since food products are reformulated by manufacturers, nutrient values in the apps may be outdated [25]. The continuous update of large food product databases remains a key challenge for diet-tracking apps [2,22]. Furthermore, the energy and nutrient contents of tonjiru were considerably overestimated in all the apps, indicating the difficulty in estimating the nutrient content of mixed dishes. Given that the Japanese diet consists primarily of a variety of mixed dishes [47], the misestimation of energy and nutrient intake by the apps may be derived mainly from the difference in the nutrient content of mixed dishes between the FCD of apps and those actually consumed.

The FiNC, Calomiru, and Mogutan apps did not provide all the information sources of nutrient values. Moreover, although some apps calculated sugar intake, the type of sugar that the app estimated was unclear. As a result, sugar intake provided by the apps differed considerably. Previous studies have also reported that information on the development process of apps or the source of nutrient values 
was not available for many commercial apps $[7,23,30]$. The lack of transparency in the information may hinder the use of diet-tracking apps in nutritional research and practice.

\subsection{Portion Size Estimation}

Portion size estimation is important since misreporting of the consumed amount is a major source of measurement error when assessing dietary intake [44,48-50]. In this study, all apps estimated the amount of food consumed based on the relative portion size entered by users. However, methods to record the relative portion size differed among apps. While most apps allowed minor adjustments of the relative portion, Mogutan offered only three categories of food portion size and FiNC required entry of the relative portion size in a unit of $10 \%$. Therefore, the exact amount of food could not always be entered in these apps. However, since the FiNC showed the standard weight for all food items, the best approximation of the relative portion size was entered based on the grams of foods written in the DR. This may explain the agreement of estimates in FiNC with the DR. Meanwhile, the other apps did not show the weight or picture of the standard portion for most food items, making it difficult to estimate the relative portion size. Noticeably, MyFitnessPal had the option to enter the quantity of foods based on units of grams or milliliters. Although accurate recording of the food amount appears to be useful, entering the precise amount of food or beverages may not always be easy, at least for some users [21,44]. Therefore, showing portion size images or household measurement units for each food item would help improve portion size estimation [44].

\subsection{Other Features}

Other app features related to usability may affect how accurately users input their food intake into apps. For instance, as the Japanese language is composed of three types of characters (hiragana, katakana, and kanji), searching for food names by different spellings produced different results in most apps. In addition, since MyFitnessPal supports 20 languages, searches in Japanese sometimes resulted in foods written in other languages, such as Chinese and English. This may make it difficult to find an exact food from a large database. Moreover, although users generally prefer large food databases, identifying correct foods from numerous food choices is complicated [44,51]. Therefore, easy-to-navigate food databases, in which the number of food entries is not overwhelming, are needed to facilitate accurate food selection [18]. In any case, to use these apps in research, more validation studies in a free-living setting are needed.

\subsection{Limitations}

Several methodological limitations of this study warrant mention. First, because our search was restricted to popular apps available for free in the Apple iTunes store, there may have been other relevant paid apps or apps distributed at other stores such as Google Play [34]. Our search strategy was based on the fact that free apps are widely used with many downloads [25,52], that the iPhone has $59.8 \%$ of the market share in Japan in 2019 [53], and that the Apple store and Google play store offerings overlap [19]. Second, we did not evaluate the actual use of apps in a free-living setting. Since dietary data were entered into the app by the author, who was a registered dietitian, the selection of foods may be more accurate compared to general users. In addition, we entered the amount of foods and beverages based on the weighed amount recorded in the DR, whereas general users may be more likely to enter portion size based on a rough estimate rather than based on weighing the amount of foods or beverages. Furthermore, the accuracy of data input may also be influenced by personal characteristics of users, such as technology literacy and personal preference [51]. Given that this study design in theory eliminated these biases involved with user entry, the concordance of estimates between the apps and DR may have been overestimated. Therefore, validation studies among free-living users are required to investigate the accuracy of estimates from apps more rigorously. Third, the input of dietary data was conducted by a single researcher and was not verified by others due to the limited number of researchers. This may have resulted in input errors and eventually misestimation of the 
apps' ability. The input of dietary data by more than one person independently would be desirable for a more rigorous evaluation. Fourth, dietary data used were collected from healthy volunteers who were not randomly selected. Moreover, we assessed only thirty dietary data records, mainly due to time constraint, although the sample size was calculated based on a previous study. Therefore, the selection of foods or the amount may not be representative, which may have affected the results of the evaluation of each app. Hence, although the sample size was sufficient to examine our research question, the results should be confirmed based on a larger number of data entries to increase the reliability of the findings. Finally, we did not evaluate the accuracy of estimates provided by the barcode scanner or automated image analysis of apps. The evaluation of these technologies to make food entry easier and quicker would be of interest in further studies.

\section{Conclusions}

In conclusion, this study investigated the features and abilities to assess dietary intake among the five popular diet-tracking apps in Japan. Each app varied in the ability to estimate nutrient intake among apps, which may be explained by the difference in app features, such as FCDs and the method to input the information regarding food intake. Although estimates from some apps were comparable to those from the paper-based DR, the results should be interpreted with caution due to the methodological limitations of this study. This study contributes to a better understanding of the ability of diet-tracking mobile apps for clinicians and researchers as well as an improved development of these types of apps. The finding reinforces the need for evaluation of diet-tracking apps to explore their potential use in nutrition research and practice. In particular, validation studies where users input their own food intake in free-living settings are needed to evaluate the ability of dietary-tracking apps more rigorously.

Supplementary Materials: The following are available online at http://www.mdpi.com/2072-6643/12/11/3327/s1, Figure S1: Bland-Altman plots assessing the agreement of the estimated protein intake between a paper-based dietary record (DR) and each application (APP) in 30 Japanese adults, Figure S2: Bland-Altman plots assessing the agreement of the estimated fat intake between a paper-based dietary record (DR) and each application (APP) in 30 Japanese adults, Figure S3: Bland-Altman plots assessing the agreement of the estimated carbohydrate intake between a paper-based dietary record (DR) and each application (APP) in 30 Japanese adults, Table S1: Basic information of the five diet-tracking applications included in this study, Table S2: Characteristics of the two validation studies for MyFitnessPal.

Author Contributions: Conceptualization, N.S. and K.M.; methodology, N.S. and K.M.; formal analysis, N.S. and K.M.; investigation, N.S.; data curation, N.S.; writing—original draft preparation, N.S. and K.M.; writing-review and editing, N.S. and K.M.; visualization, N.S. and K.M.; supervision, K.M.; project administration, N.S. and K.M. All authors have read and agreed to the published version of the manuscript.

Funding: This research was funded by the Ministry of Health, Labor, and Welfare, Japan, grant number H23-jyunkankitou (seishuu)-ippan-001.

Acknowledgments: The authors thank Keiko Asakura, Shizuko Masayasu, and Satoshi Sasaki for data collection.

Conflicts of Interest: The authors declare no conflict of interest. The funders had no role in the design of the study; in the collection, analyses, or interpretation of data; in the writing of the manuscript; or in the decision to publish the results.

\section{References}

1. Shriver, B.J.; Roman-Shriver, C.R.; Long, J.D. Technology-based methods of dietary assessment: Recent developments and considerations for clinical practice. Curr. Opin. Clin. Nutr. Metab. Care 2010, 13, 548-551. [CrossRef]

2. Cade, J.E. Measuring diet in the 21st century: Use of new technologies. Proc. Nutr. Soc. 2017, 76, $276-282$. [CrossRef] [PubMed]

3. Fallaize, R.; Franco, R.Z.; Pasang, J.; Hwang, F.; Lovegrove, J.A. Popular nutrition-related mobile apps: An agreement assessment against a UK reference method. J. Med. Internet Res. 2019, 21, 1-13. [CrossRef] [PubMed] 
4. Sharp, D.B.; Allman-Farinelli, M. Feasibility and validity of mobile phones to assess dietary intake. Nutrition 2014, 30, 1257-1266. [CrossRef] [PubMed]

5. Illner, A.K.; Freisling, H.; Boeing, H.; Huybrechts, I.; Crispim, S.P.; Slimani, N. Review and evaluation of innovative technologies for measuring diet in nutritional epidemiology. Int. J. Epidemiol. 2012, 41, 1187-1203. [CrossRef] [PubMed]

6. Ngo, J.; Engelen, A.; Molag, M.; Roesle, J.; García-Segovia, P.; Serra-Majem, L. A review of the use of information and communication technologies for dietary assessment. Br. J. Nutr. 2009, 101, S102-S112. [CrossRef]

7. Eldridge, A.L.; Piernas, C.; Illner, A.-K.; Gibney, M.J.; Gurinović, M.A.; de Vries, J.H.M.; Cade, J.E. Evaluation of new technology-based tools for dietary intake-An ILSI Europe Dietary Intake and Exposure Task Force evaluation. Nutrients 2018, 11, 55. [CrossRef]

8. Turner-McGrievy, G.M.; Beets, M.W.; Moore, J.B.; Kaczynski, A.T.; Barr-Anderson, D.J.; Tate, D.F. Comparison of traditional versus mobile app self-monitoring of physical activity and dietary intake among overweight adults participating in an mHealth weight loss program. J. Am. Med. Inform. Assoc. 2013, 20, 513-518. [CrossRef]

9. Chen, J.; Lieffers, J.; Bauman, A.; Hanning, R.; Allman-Farinelli, M. The use of smartphone health apps and other mobile health (mHealth) technologies in dietetic practice: A three country study. J. Hum. Nutr. Diet. 2017, 30, 439-452. [CrossRef]

10. Sauceda, A.; Frederico, C.; Pellechia, K.; Starin, D. Results of the Academy of Nutrition and Dietetics' Consumer Health Informatics Work Group's 2015 Member App Technology Survey. J. Acad. Nutr. Diet. 2016, 116, 1336-1338. [CrossRef]

11. Martin, C.K.; Han, H.; Coulon, S.M.; Allen, H.R.; Champagne, C.M.; Anton, S.D. A novel method to remotely measure food intake of free-living individuals in real time: The remote food photography method. Br. J. Nutr. 2009, 101, 446-456. [CrossRef]

12. Carter, M.C.; Burley, V.J.; Nykjaer, C.; Cade, J.E. Adherence to a smartphone application for weight loss compared to website and paper diary: Pilot randomized controlled trial. J. Med. Internet Res. 2013, 15, 1-17. [CrossRef]

13. Ipjian, M.L.; Johnston, C.S. Smartphone technology facilitates dietary change in healthy adults. Nutrition 2017, 33, 343-347. [CrossRef] [PubMed]

14. Ambrosini, G.L.; Hurworth, M.; Giglia, R.; Trapp, G.; Strauss, P. Feasibility of a commercial smartphone application for dietary assessment in epidemiological research and comparison with 24-h dietary recalls. Nutr. J. 2018, 17, 5. [CrossRef]

15. Hutchesson, M.J.; Rollo, M.E.; Callister, R.; Collins, C.E. Self-monitoring of dietary intake by young women: Online food records completed on computer or smartphone are as accurate as paper-based food records but more acceptable. J. Acad. Nutr. Diet. 2015, 115, 87-94. [CrossRef] [PubMed]

16. Khazen, W.; Jeanne, J.F.; Demaretz, L.; Schäfer, F.; Fagherazzi, G. Rethinking the use of mobile apps for dietary assessment in medical research. J. Med. Internet Res. 2020, 22, e15619. [CrossRef] [PubMed]

17. Bardus, M.; van Beurden, S.B.; Smith, J.R.; Abraham, C. A review and content analysis of engagement, functionality, aesthetics, information quality, and change techniques in the most popular commercial apps for weight management. Int. J. Behav. Nutr. Phys. Act. 2016, 13, 1-9. [CrossRef]

18. Tang, J.; Abraham, C.; Stamp, E.; Greaves, C. How can weight-loss app designers' best engage and support users? A qualitative investigation. Br. J. Health Psychol. 2015, 20, 151-171. [CrossRef]

19. Griffiths, C.; Harnack, L.; Pereira, M.A. Assessment of the accuracy of nutrient calculations of five popular nutrition tracking applications. Public Health Nutr. 2018, 21, 1495-1502. [CrossRef]

20. Carter, M.C.; Burley, V.J.; Nykjaer, C.; Cade, J.E. My Meal Mate (MMM): Validation of the diet measures captured on a smartphone application to facilitate weight loss. Br. J. Nutr. 2013, 109, 539-546. [CrossRef]

21. Teixeira, V.; Voci, S.M.; Mendes-Netto, R.S.; da Silva, D.G. The relative validity of a food record using the smartphone application MyFitnessPal. Nutr. Diet. 2018, 75, 219-225. [CrossRef] [PubMed]

22. Maringer, M.; Wisse-Voorwinden, N.; Van'T Veer, P.; Geelen, A. Food identification by barcode scanning in the Netherlands: A quality assessment of labelled food product databases underlying popular nutrition applications. Public Health Nutr. 2019, 22, 1215-1222. [CrossRef] [PubMed]

23. Braz, V.N.; de Moraes Lopes, M.H.B. Evaluation of mobile applications related to nutrition. Public Health Nutr. 2019, 22, 1209-1214. [CrossRef] [PubMed] 
24. Chen, J.; Cade, J.E.; Allman-Farinelli, M. The most popular smartphone apps for weight loss: A quality assessment. JMIR mHealth uHealth 2015, 3, e104. [CrossRef] [PubMed]

25. Ferrara, G.; Kim, J.; Lin, S.; Hua, J.; Seto, E. A focused review of smartphone diet-tracking apps: Usability, functionality, coherence with behavior change theory, and comparative validity of nutrient intake and energy estimates. JMIR mHealth uHealth 2019, 7, 1-16. [CrossRef]

26. Rangan, A.M.; Connor, S.O.; Giannelli, V.; Yap, M.L.H.; Ming, L.; Hon, B.C.S.; Roy, R.; Chun, J.; Louie, Y.; Hebden, L.; et al. Electronic Dietary Intake Assessment (e-DIA): Comparison of a mobile phone digital entry app for dietary data collection with 24-hour dietary recalls. JMIR mHealth $u$ Health 2015, 3, e98. [CrossRef]

27. iTunes Search API. Available online: https://affiliate.itunes.apple.com/resources/documentation/itunes-storeweb-service-search-api/ (accessed on 4 August 2020).

28. Nikolaou, C.K.; Lean, M.E.J. Mobile applications for obesity and weight management: Current market characteristics. Int. J. Obes. 2017, 41, 200-202. [CrossRef]

29. Li, Y.; Ding, J.; Wang, Y.; Tang, C.; Zhang, P. Nutrition-related mobile apps in the China app store: Assessment of functionality and quality. JMIR mHealth uHealth 2019, 7, 1-11. [CrossRef]

30. Maringer, M.; Van'T Veer, P.; Klepacz, N.; Verain, M.C.D.; Normann, A.; Ekman, S.; Timotijevic, L.; Raats, M.M.; Geelen, A. User-documented food consumption data from publicly available apps: An analysis of opportunities and challenges for nutrition research. Nutr. J. 2018, 17, 59. [CrossRef]

31. Schoeppe, S.; Alley, S.; Rebar, A.L.; Hayman, M.; Bray, N.A.; Van Lippevelde, W.; Gnam, J.P.; Bachert, P.; Direito, A.; Vandelanotte, C. Apps to improve diet, physical activity and sedentary behaviour in children and adolescents: A review of quality, features and behaviour change techniques. Int. J. Behav. Nutr. Phys. Act. 2017, 14, 1-10. [CrossRef]

32. Xu, W.; Liu, Y. mHealthApps: A repository and database of mobile health apps. JMIR mHealth uHealth 2015, 3, e28. [CrossRef]

33. Zaidan, S.; Roehrer, E. Popular mobile phone apps for diet and weight loss: A content analysis. JMIR mHealth uHealth 2016, 4, e80. [CrossRef]

34. Schumer, H.; Amadi, C.; Joshi, A. Evaluating the dietary and nutritional apps in the google play store. Healthc. Inform. Res. 2018, 24, 38-45. [CrossRef]

35. Franco, R.Z.; Fallaize, R.; Lovegrove, J.A.; Hwang, F. Popular nutrition-related mobile apps: A feature assessment. JMIR mHealth uHealth 2016, 4, e85. [CrossRef]

36. Asakura, K.; Uechi, K.; Sasaki, Y.; Masayasu, S.; Sasaki, S. Estimation of sodium and potassium intakes assessed by two $24 \mathrm{~h}$ urine collections in healthy Japanese adults: A nationwide study. Br. J. Nutr. 2014, 112, 1195-1205. [CrossRef] [PubMed]

37. Asakura, K.; Uechi, K.; Masayasu, S.; Sasaki, S. Sodium sources in the Japanese diet: Difference between generations and sexes. Public Health Nutr. 2016, 19, 2011-2023. [CrossRef]

38. Shinozaki, N.; Murakami, K.; Masayasu, S.; Sasaki, S. Development and simulated validation of a dish composition database for estimating food group and nutrient intakes in Japan. Public Health Nutr. 2019, 22, 2367-2380. [CrossRef] [PubMed]

39. Council for Science and Technology; Ministry of Education, Culture, Sports Science and Technology, Japan. Standard Tables of Food Composition in Japan 2015, 7th revised ed.; Official Gazette Co-Operation of Japan: Tokyo, Japan, 2015.

40. Whybrow, S.; Horgan, G.W.; Macdiarmid, J.I. Self-reported food intake decreases over recording period in the National Diet and Nutrition Survey. Br. J. Nutr. 2020, 124, 586-590. [CrossRef]

41. Mela, D.J.; MWoolner, E. Perspective: Total, added, or free? What kind of sugars should we be talking about? Adv. Nutr. 2018, 9, 63-69. [CrossRef] [PubMed]

42. Bland, J.M.; Altman, D.G. Comparing methods of measurement: Why plotting difference against standard method is misleading. Lancet 1995, 346, 1085-1087. [CrossRef]

43. Giavarina, D. Understanding Bland Altman analysis. Biochem. Med. 2015, 25, 141-151.

44. Chen, J.; Berkman, W.; Bardouh, M.; Ng, C.Y.K.; Allman-Farinelli, M. The use of a food logging app in the naturalistic setting fails to provide accurate measurements of nutrients and poses usability challenges. Nutrition 2019, 57, 208-216.

45. Dietary Reference Intake for Japanese. 2020. Available online: https:/www.mhlw.go.jp/content/10904750/ 000586553.pdf (accessed on 3 September 2020). 
46. Food Labeling Act. Available online: https://www.caa.go.jp/policies/policy/food_labeling/food_labeling_act/ (accessed on 15 September 2020).

47. Shinozaki, N.; Murakami, K.; Masayasu, S.; Sasaki, S. Validity of a dish composition database for estimating protein, sodium and potassium intakes against $24 \mathrm{~h}$ urinary excretion: Comparison with a standard food composition database. Public Health Nutr. 2020, 23, 1297-1306. [PubMed]

48. Amoutzopoulos, B.; Page, P.; Roberts, C.; Roe, M.; Cade, J.; Steer, T.; Baker, R.; Hawes, T.; Galloway, C.; Yu, D.; et al. Portion size estimation in dietary assessment: A systematic review of existing tools, their strengths and limitations. Nutr. Rev. 2020, 78, 885-900. [PubMed]

49. Beasley, J.; Riley, W.T.; Jean-Mary, J. Accuracy of a PDA-based dietary assessment program. Nutrition 2005, 21, 672-677. [PubMed]

50. Hernández, T.; Wilder, L.; Kuehn, D.; Rubotzky, K.; Moser-Veillon, P.; Godwin, S.; Thompson, C.; Wang, C. Portion size estimation and expectation of accuracy. J. Food Compos. Anal. 2006, 19, 14-21.

51. Lieffers, J.R.L.; Arocha, J.F.; Grindrod, K.; Hanning, R.M. Experiences and perceptions of adults accessing publicly available nutrition behavior-change mobile apps for weight management. J. Acad. Nutr. Diet. 2018, 118, 229-239.e3.

52. Azar, K.M.J.; Lesser, L.I.; Laing, B.Y.; Stephens, J.; Aurora, M.S.; Burke, L.E.; Palaniappan, L.P. Mobile applications for weight management: Theory-based content analysis. Am. J. Prev. Med. 2013, 45, 583-589.

53. White Paper Information and Communications in Japan. Available online: https://www.soumu.go.jp/ johotsusintokei/whitepaper/ja/r02/pdf/index.html (accessed on 26 October 2020).

Publisher's Note: MDPI stays neutral with regard to jurisdictional claims in published maps and institutional affiliations.

(C) 2020 by the authors. Licensee MDPI, Basel, Switzerland. This article is an open access article distributed under the terms and conditions of the Creative Commons Attribution (CC BY) license (http://creativecommons.org/licenses/by/4.0/). 